Check for updates

Cite this: Phys. Chem. Chem. Phys., 2018, 20, 6805

Received 16th November 2017 Accepted 19th February 2018

DOI: $10.1039 / c 7 c p 07738 f$

rsc.li/pccp

\section{Collective thermal transport in pure and alloy semiconductors}

\author{
Pol Torres, (D) *a Amr Mohammed, ${ }^{\mathrm{b}}$ Àlvar Torelló, ${ }^{\mathrm{a}}$ Javier Bafaluy, ${ }^{a}$ \\ Juan Camacho, (D) a Xavier Cartoixà, ${ }^{c}$ Ali Shakouri ${ }^{b}$ and F. Xavier Alvarez ${ }^{a}$
}

Conventional models for predicting thermal conductivity of alloys usually assume a pure kinetic regime as alloy scattering dominates normal processes. However, some discrepancies between these models and experiments at very small alloy concentrations have been reported. In this work, we use the full first principles kinetic collective model (KCM) to calculate the thermal conductivity of $\mathrm{Si}_{1-x} \mathrm{Ge}_{x}$ and $\ln _{x} \mathrm{Ga}_{1-x}$ As alloys. The calculated thermal conductivities match well with the experimental data for all alloy concentrations. The model shows that the collective contribution must be taken into account at very low impurity concentrations. For higher concentrations, the collective contribution is suppressed, but normal collisions have the effect of significantly reducing the kinetic contribution. The study thus shows the importance of the proper inclusion of normal processes even for alloys for accurate modeling of thermal transport. Furthermore, the phonon spectral distribution of the thermal conductivity is studied in the framework of KCM, providing insights to interpret the superdiffusive regime introduced in the truncated Lévy flight framework.

Over the past years, thermal conductivity of a large number of bulk materials has been accurately calculated using phenomenological $^{1,2}$ and $a b$ initio $^{3-6}$ techniques. Proper inclusion of normal $(\mathrm{N})$ processes (i.e. momentum conserving phonon collisions) in the calculations has played a key role. This procedure has allowed probing new transport regimes like hydrodynamic heat flow or collective behavior in samples where $\mathrm{N}$ processes are important. $^{7,8}$

Despite of the level of accuracy achieved, there are still some open questions. On one hand, although it is well known that size effects reduce the thermal conductivity, this reduction can not be fully explained from pure kinetic models, which only account for the resisitve scattering mechanisms (Umklapp (U),

\footnotetext{
${ }^{a}$ Departament de Física, Universitat Autònoma de Barcelona, 08193 Bellaterra, Catalonia, Spain.E-mail: pol.torres@uab.cat

${ }^{b}$ Birck Nanotechnology Center, Purdue University, West Lafayette, Indiana 47907, USA

${ }^{c}$ Departament d'Enginyeria Electrònica, Universitat Autònoma de Barcelona, 08193 Bellaterra, Catalonia, Spain
}

boundary, mass defect), without the introduction of new fitting parameters. Current models require modifying the relaxation times expressions typically used for the bulk material or including new scattering mechanisms. ${ }^{9,10}$ On the other hand, it is widely known that alloying is also a successful strategy for thermal conductivity reduction. However, precise calculations of thermal transport at small alloy concentrations remain unexplored. All this hinders the interpretation of spectral information in terms of characteristic lengths and times resolved in ultrafast heating phenomena observed in experiments such as Time Domain and Frequency Domain Thermoreflectance (TDTR/FDTR). ${ }^{11}$

The Kinetic Collective Model (KCM $)^{1,2}$ offers a natural framework to understand the role of $\mathrm{N}$ processes in thermal transport. In this model, a collective mechanism is introduced as a result of the $\mathrm{N}$ scattering processes, where all the phonons share a unique value for the resistive relaxation time. ${ }^{1,2,6}$ Unlike Relaxation Time Approximation (RTA) models, KCM does not treat the $\mathrm{N}$ processes as resistive (R) processes. Instead, KCM uses $\mathrm{N}$ processes to calculate the relative weight between collective and kinetic contributions. This approach is in accordance with Peierls' initial work where the non-resistive nature of $\mathrm{N}$ scattering was demonstrated. ${ }^{12}$ While other recently developed methods, such as full-BTE solutions (iterative method, ${ }^{13}$ the relaxon solution $^{14}$ and the direct solution ${ }^{15}$ ), also capture the effects of collective contributions, obtaining physical insight from the computed results is harder due to the heavy numerical character of these approaches. In addition, it is hard for these methods to go from bulk to the nanoscale. ${ }^{6}$ On the other hand, the formalism underlying KCM makes it easier to develop more accurate predictions of the thermal conductivity and how it is enhanced or reduced at small scales or lower dimensions.

In the present work, we focus on the role played by $\mathrm{N}$ scattering on alloys. On the one hand, we show that some difficulties in predicting pure and alloy experimental thermal conductivities with the same model are explained by the reduction of the collective contribution as impurity increases due to the enhancement of resistive effects caused by alloying. In these cases, trying to fit 
the thermal conductivity with RTA for high alloy concentrations, the value for pure materials is underestimated, $\uparrow^{16}$ as the collective contribution is neglected. On the other hand, even when the collective contribution is negligible and thermal transport is dominated by resistive collisions, $\mathrm{N}$ scattering is shown to significantly slow down kinetic transport. We also propose that the presence of a collective regime could offer a basis to explain the significant differences in the frequency behaviour between alloy and pure materials in frequency domain termoreflectance (FDTR) experiments. ${ }^{11}$

We study two well-known and extensively used thermoelectric and optoelectronic alloys, $\mathrm{Si}_{1-x} \mathrm{Ge}_{x}$ and $\mathrm{In}_{x} \mathrm{Ga}_{1-x} \mathrm{As}$. Such materials have a diamond/zincblende-like structure, with a $\mathrm{Si}_{1-x} \mathrm{Ge}_{x}$ and $\mathrm{In}_{x} \mathrm{Ga}_{1-x}$ atom in the $(0,0,0)$ position and $\mathrm{Si}_{1-x} \mathrm{Ge}_{x}$ and As at $(1 / 2,1 / 2,1 / 2)$ respectively. First principles KCM calculations at several germanium and indium concentrations $x$ are performed in each case.

The total lattice thermal conductivity $\kappa_{\mathrm{T}}$ is formulated in KCM model as the sum of the kinetic $\kappa_{\mathrm{k}}$ and the collective $\kappa_{\mathrm{c}}$ terms $\left(\kappa_{\mathrm{T}}=\kappa_{\mathrm{k}}+\kappa_{\mathrm{c}}\right)$ : 6,18

$$
\begin{gathered}
\kappa_{\mathrm{k}}=(1-\Sigma) \int C_{\mathrm{v}} v^{2} \tau_{\mathrm{k}} D \mathrm{~d} \omega \\
\kappa_{\mathrm{c}}=\Sigma \cdot F \int C_{\mathrm{v}} v^{2} \tau_{\mathrm{c}} D \mathrm{~d} \omega,
\end{gathered}
$$

where $C_{\mathrm{v}}(\omega)$ and $v(\omega)$ are the mode heat capacity and velocity respectively, $\tau_{\mathrm{k}}\left(L_{\mathrm{eff}}, \omega, T\right)$ and $\tau_{\mathrm{c}}(T)$ denote the kinetic and collective mean free times (MFT) and $D(\omega)$ the density of states (DOS) for each mode. Details of how $\tau_{\mathrm{k}}$ and $\tau_{\mathrm{c}}$ are calculated can be found elsewhere. ${ }^{1,2,6} L_{\text {eff }}$ is the effective width of the sample, which depends on its geometry. The $\Sigma\left(L_{\text {eff }}, T\right)$ factor, determined by the distribution averaged values of normal and kinetic scattering MFT, $\left\langle\tau_{\mathrm{N}}\right\rangle(T)$ and $\left\langle\tau_{\mathrm{k}}\right\rangle(T)$ :

$$
\Sigma=\frac{1}{\left(1+\left\langle\tau_{\mathrm{N}}\right\rangle /\left\langle\tau_{\mathrm{k}}\right\rangle\right)} \in[0,1]
$$

weighs the contribution of the kinetic and collective terms to thermal transport. The form factor $F\left(L_{\text {eff }}, T\right)$ takes into account hydrodynamic reduction of heat flux due to collective boundary scattering, similar to the Poiseuille flow. Detailed expressions for all these terms are given elsewhere. ${ }^{1,2,6}$ It is important to notice that the kinetic contribution $\kappa_{\mathrm{k}}$ of eqn (1) is not the pure kinetic value $\hat{k}_{\mathrm{k}}=\int C_{\mathrm{v}} v^{2} \tau_{\mathrm{k}} D \mathrm{~d} \omega$, since it is corrected through the $(1-\Sigma)$ factor as a consequence of normal collisions, i.e. $k_{\mathrm{k}}=(1-\Sigma) \hat{k}_{\mathrm{k}}$.

In order to compute the properties of the alloys, the phonon dispersion relations and the relaxation times are needed. To obtain the dispersion relations for a stoichiometry $x$, we perform lattice dynamics calculations within the Virtual Crystal Approximation (VCA) in a first principles framework. ${ }^{19}$ Calculations were done with the Quantum ESPRESSO package, ${ }^{20}$ under the Density Functional Theory (DFT), ${ }^{21,22}$ and the Local Density Approximation (LDA) in the parameterization of Perdew and Zunger. ${ }^{23}$ Plane waves from norm-conserving pseudopotentials of the von Barth-Car type $\mathrm{e}^{24,25}$ were cut off at an energy of 60 Rydberg.
Born effective charges have been considered for the polar compounds. At each composition $x$, the lattice parameter was adjusted until the pressure was less than 0.1 kbar. Solution of the ensuing dynamical matrix provides the dispersion relations and transport parameters. As the shape of the dispersion relations depends on the crystal structure and atomic masses, their shape will be very similar to that of their constituent materials. ${ }^{26,27}$

For the above materials, four different relaxation times should be considered. The resisitve scattering terms, which contribute to the total resistivity, are arranged in the kinetic MFP using the Mathiessen's rule

$$
\frac{1}{\tau_{\mathrm{k}}}=\frac{1}{\tau_{\mathrm{I}}}+\frac{1}{\tau_{\mathrm{B}}}+\frac{1}{\tau_{\mathrm{U}}},
$$

where $\tau_{\mathrm{B}}\left(L_{\mathrm{eff}}, \omega\right)$ is the boundary term, $\tau_{\mathrm{U}}(\omega, T)$ the umklapp relaxation time and $\tau_{\mathrm{I}}(\omega)$ the impurity term. The fourth element is the $\mathrm{N}$ scattering term $\tau_{\mathrm{N}}$, that is included just in the $\Sigma$ factor. All these terms depend on the alloy fraction $x$. For boundary scattering in the kinetic term we use Casimir's expression ${ }^{32}$

$$
\tau_{\mathrm{B}}=L_{\mathrm{eff}} / v,
$$

where $L_{\text {eff }}=d_{\text {wire }}$ is the diameter for wires, $L_{\text {eff }}=2.25 h$ for films, where $h$ is the thickness, and $L_{\text {eff }}=1.12 \sqrt{l_{1} l_{2}}$ for rods, where $l_{1}$ and $l_{2}$ are the rod thickness and width. ${ }^{1}$

Umklapp and normal scattering rates are computed from first principles calculations for natural silicon and gallium arsenide. For $\mathrm{Si}_{1-x} \mathrm{Ge}_{x}$ alloys, such calculations have been carried in the VCA for $x=0.0025,0.004,0.01,0.04,0.1,0.18,0.2,0.4,0.5$, 0.82 and for $\operatorname{In}_{x} \mathrm{Ga}_{1-x} \mathrm{As}$ at $x=0.01,0.15,0.3,0.53$ compositions. Interpolation has been used in the latter case for smaller and intermediate concentrations.

Regarding impurity scattering, the mass defect term describes the variability in isotopic abundance for single-species crystals, but in alloys it should also account for the variability in the force and lattice constants. Thus, including these three factors, the total alloy relaxation time can be expressed as: ${ }^{33}$

$$
\tau_{\mathrm{I}}^{-1}=\frac{\pi}{6} V S^{2} \omega^{2} D
$$

where as done by Capinski et al. ${ }^{34}$ instead of the Debye approximation, we use the DOS obtained from the full dispersion relations at stoichiometry $x$. The variance term $S$ is:

$$
S_{\mathrm{i}}^{2}=\frac{1}{12} \Gamma_{M}^{2}+\left(\frac{1}{\sqrt{6}} \Gamma_{v^{2}}-\sqrt{\frac{2}{3}} Q \gamma \Gamma_{R}\right)^{2}
$$

for each impurity, where $\Gamma_{\alpha}$ is the coefficient of variance of $(M)$ mass, $\left(v^{2}\right)$ squared velocity or $(R)$ impurity radius. $Q=4.2$ is a factor depending on the geometry of the impurity (substitution in this case) and $\gamma$ is the Grüneisen parameter calculated from first principles. As expected, the second and third terms reduce to zero for pure materials.

Fig. 1 shows the KCM prediction of the thermal conductivity and the weighting factor $\Sigma$ for $\mathrm{Si}_{1-x} \mathrm{Ge}_{x}$ and $\mathrm{In}_{x} \mathrm{Ga}_{1-x} \mathrm{As}$ rods at $300 \mathrm{~K}$. One observes that theoretical predictions (black lines) agree with experimental data for $\mathrm{Si}_{1-x} \mathrm{Ge}_{x}$. For $\mathrm{In}_{x} \mathrm{Ga}_{1-x} \mathrm{As}$ we note inconsistencies between old published bulk experimental 
a)

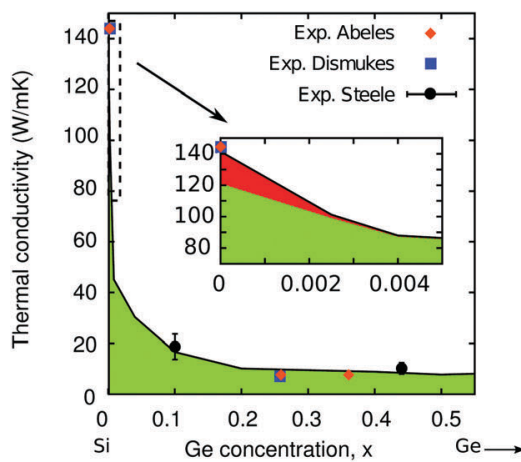

b)

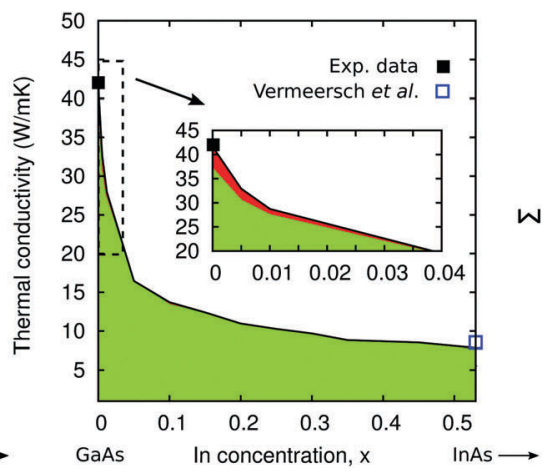

c)

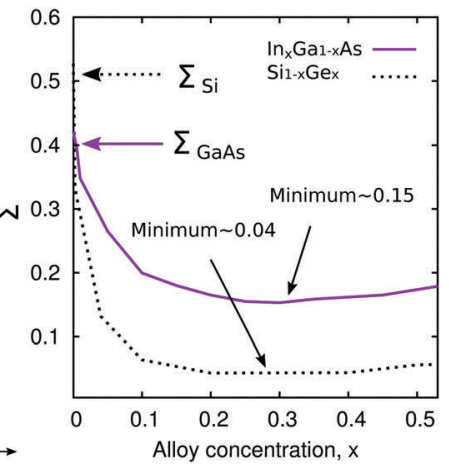

Fig. 1 Thermal conductivity of $L_{\text {eff }}=7 \mathrm{~mm}$ rods in terms of alloy concentration $x$ at $300 \mathrm{~K}$ for (a) $\mathrm{Si}_{1-x} \mathrm{Ge}_{x}$ and (b) $\ln _{x} G a_{1-x} A s$. Green and red filled zones denote the kinetic and collective contributions to the thermal conductivity respectively. Black solid lines denote the total thermal conductivity. Insets represent magnifications at low impurity concentrations. (c) Switching factor $\Sigma$ corresponding to (a) and (b) showing the transition to a kinetic-dominant regime as impurity increases. Experimental data for $\mathrm{Si}_{1-x} \mathrm{Ge}_{x}$ are from ref. 28-30. GaAs experimental datum is taken from ref. 17 and for $\ln _{0.53} \mathrm{Ga}_{0.47} \mathrm{~A}$ from ref. 31 .

data, ${ }^{28,35}$ reporting values smaller than $5 \mathrm{~W} \mathrm{~m}^{-1} \mathrm{~K}^{-1}$, and recent $1.6 \mu \mathrm{m}$ thin films measurements of $5.5 \mathrm{~W} \mathrm{~m}^{-1} \mathrm{~K}^{-1}$ for $\mathrm{In}_{0.53} \mathrm{Ga}_{0.47} \mathrm{As}$ at $300 \mathrm{~K} .{ }^{36}$ Therefore bulk values are expected to be higher than $5.5 \mathrm{~W} \mathrm{~m}^{-1} \mathrm{~K}^{-1}$. Note that collective thermal transport (red region) is only important for very pure materials close to $x=0$, being mostly destroyed with impurity fractions as low as $0.4 \%$ and $4 \%$ for $\mathrm{Si}_{1-x} \mathrm{Ge}_{x}$ and $\mathrm{In}_{x} \mathrm{Ga}_{1-x} \mathrm{As}$ respectively.

From the insets it can be appreciated that although the kinetic contribution can describe most of the concentration range, it is not able to explain the conductivity near the pure region $x \sim 0$, where the collective term contributes up to a $10 \%$ of the thermal conductivity. ${ }^{16}$ It is the correct treatment of $\mathrm{N}$ processes, as done by KCM or iterative and full solution methods, that provides good predictions at all concentrations. These results show that a proper description of the collective contribution is necessary to understand the large drop in thermal conductivity at small impurity concentrations. In the case of $\operatorname{In}_{x} \mathrm{Ga}_{1-x} \mathrm{As}$, it is visible that the reduction of the collective contribution when the alloy concentration is increased is not as sharp as that found in $\mathrm{Si}_{1-x} \mathrm{Ge}_{x}$. This is a consequence of the difference of the strength of the alloy scattering in each sample: the isotopic mass variation term in $\mathrm{Si}_{1-x} \mathrm{Ge}_{x}$ alloys is several times larger than in $\operatorname{In}_{x} \mathrm{Ga}_{1-x}$ As alloys. A magnitude that quantifies these differences is the $\Sigma$ factor (Fig. 1c). For pure materials like Si and GaAs we found values of $\Sigma_{\mathrm{Si}} \sim 0.5$ and $\Sigma_{\text {GaAs }} \sim 0.4$. When the alloy concentration is increased this value goes fast below 0.1 for $\mathrm{Si}_{1-x} \mathrm{Ge}_{x}$, with a minimum of $\sim 0.04$ around $\mathrm{Si}_{0.7} \mathrm{Ge}_{0.3}$. In contrast, in the case of $\operatorname{In}_{x} \mathrm{Ga}_{1-x} \mathrm{As}$ the reduction of $\Sigma$ is smoother, decreasing from $0.4 \rightarrow 0.15$ for In concentrations going from $x=0 \rightarrow x=0.3$. Since the larger $\Sigma$ the larger the collective contribution, the sharper change of $\Sigma$ in $\mathrm{Si}_{1-x} \mathrm{Ge}_{x}$ alloys as impurity increases translates into a sharper drop in conductivity, as displayed in Fig. 1.

A second issue to be pointed out, is that as consequence of $\mathrm{N}$ scattering, not only a collective contribution to thermal conductivity does appear, but it also causes the slowdown of kinetic modes. The pure kinetic term $\hat{\kappa}_{\mathrm{k}}$ is thus reduced by a factor $(1-\Sigma)$. Since the minimum values of $\Sigma$ displayed in
Fig. 1c are 0.04 for $\mathrm{Si}_{1-x} \mathrm{Ge}_{x}$ and 0.15 for $\mathrm{In}_{x} \mathrm{Ga}_{1-x} \mathrm{As}$, this correction amounts at least a $4 \%$ and a $15 \%$, respectively. In general it will be more important in $\mathrm{In}_{x} \mathrm{Ga}_{1-x} \mathrm{As}$ than in $\mathrm{Si}_{1-x} \mathrm{Ge}_{x}$.
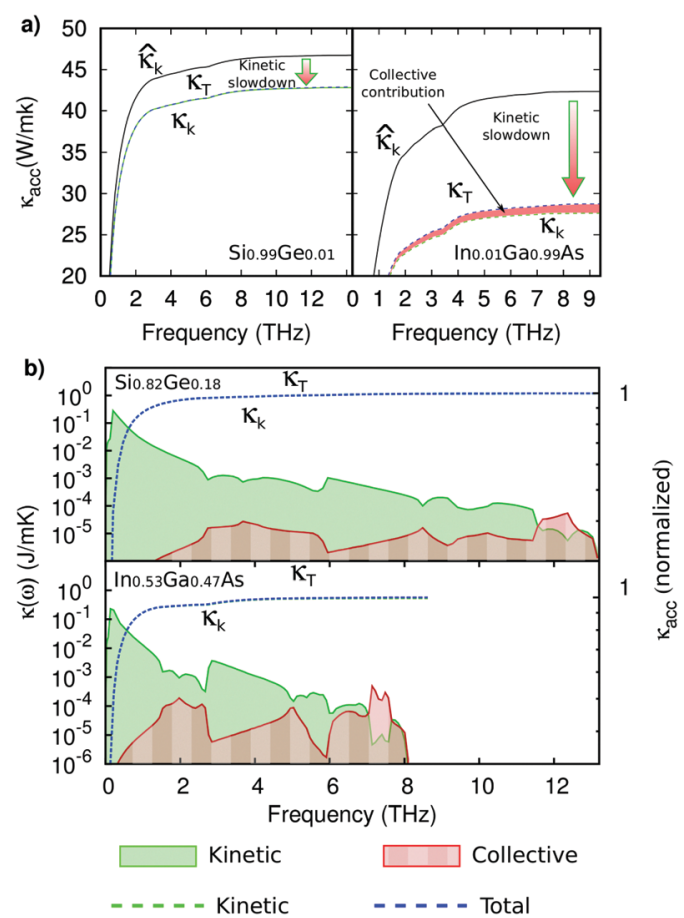

Fig. 2 (a) Thermal conductivity accumulation function (TCAF) for $\mathrm{Si}_{0.99} \mathrm{Ge}_{0.01}$ (left) and $\mathrm{In}_{0.01} \mathrm{Ga}_{0.99} \mathrm{As}$ (right) for $7 \mathrm{~mm}$ rods at $T=300 \mathrm{~K}$. (b) Thermal conductivity spectral distribution (TCSD) and TCAF in terms of frequency for $\mathrm{Si}_{0.82} \mathrm{Ge}_{0.18}$ (top) and $\mathrm{In}_{0.53} \mathrm{Ga}_{0.47} \mathrm{As}$ (bottom) for $7 \mathrm{~mm}$ rods at $T=300 \mathrm{~K}$.

Fig. 2a displays the thermal conductivity accumulation function (TCAF) for the two alloys at $x=0.01$, showing a significant slowing down of kinetic transport. This reduction of the kinetic heat transport due to $\mathrm{N}$ collisions is modelled in the classical RTA approach through the inclusion of $\mathrm{N}$ scattering 
as a resistive mechanism in the Mattiessen rule. The present model thus helps to understand why, when $\Sigma$ is small, the RTA is expected to work. Notice that KCM offers a more general framework for accounting the effects of $\mathrm{N}$ scattering, namely, the reduction of kinetic transport, and the existence of a collective heat transport.

From a microscopic point of view, collective and kinetic contributions should have very different transient behaviour. This can be shown by noticing that while each mode has a different MFP in the kinetic regime, the collective MFP is the same for all of them. In Fig. $2 b$ the phonon spectral distribution of the thermal conductivity of the two common alloy concentrations $\mathrm{Si}_{0.82} \mathrm{Ge}_{0.18}$ (top) and $\mathrm{In}_{0.53} \mathrm{Ga}_{0.47} \mathrm{As}$ (bottom) at $300 \mathrm{~K}$ and $L_{\text {eff }}=7 \mathrm{~mm}$ are shown. The green region represents the kinetic and the red the collective contribution. Notice that, differently to a pure kinetic approach, all the contribution for the collective regime that spans the whole frequency spectrum is to be assigned to a single collective mean free path $v \tau_{\mathrm{c}}{ }^{6}$ In pure materials like GaAs, $\mathrm{C}$, Ge or $\mathrm{Si}$, the collective contribution is larger than in alloys. ${ }^{6}$ This could offer an explanation of why the reduction of thermal conductivity in FDTR experiments in pure samples occurs at higher frequencies.

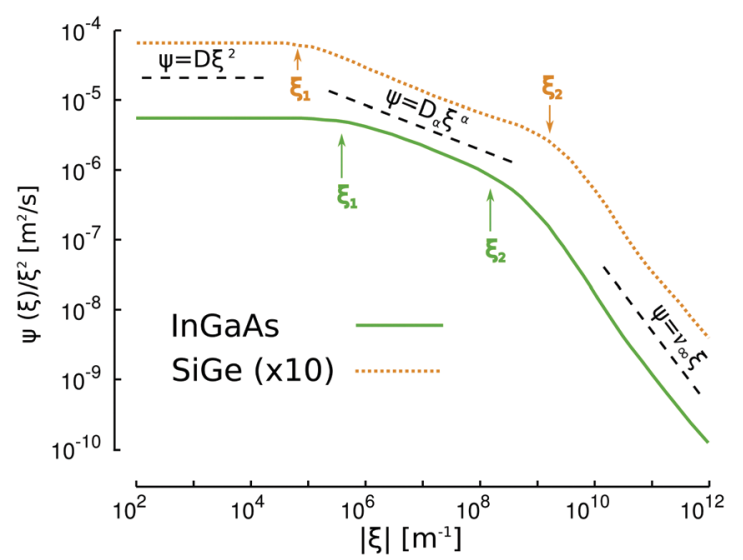

Fig. 3 Fractal diffusivity defined from $\psi(\xi) / \xi^{2}$ for $\ln _{0.53} G_{0.47} A s$ and $\mathrm{Si}_{0.82} \mathrm{Ge}_{0.18} . \mathrm{Si}_{0.82} \mathrm{Ge}_{0.18}$ curve is upscaled to ease the visualization. ${ }^{31}$ Three scaling regions with different exponents may be identified.

The transient behaviour can be explained in more detail if we combine KCM with the Truncated Lévy-flights (TL) formalism. ${ }^{31,37}$ This provides a unified description of the transition from diffusive to ballistic thermal transport in pure and alloy semiconductors. In the time domain, this transition from short to long time scales can be described by the Maxwell-Cattaneo equation: ${ }^{38}$

$$
\tau \frac{\partial^{2} T}{\partial t^{2}}+\frac{\partial T}{\partial t}=\chi \nabla^{2} T
$$

where $\tau$ is the mean characteristic time of phonon distribution and $\chi$ is the thermal diffusivity of the sample. When the inertial term is negligible $\left(\tau \frac{\partial^{2} T}{\partial t^{2}} \ll \frac{\partial T}{\partial t}\right)$ the diffusion equation is recovered, and in the opposite case $\left(\tau \frac{\partial^{2} T}{\partial t^{2}} \gg \frac{\partial T}{\partial t}\right)$ we obtain the wave equation. Despite of this clear distinction, dynamics of energy transport in actual semiconductors is more complex. Eqn (8) is valid only when a single characteristic time $\tau$ can be defined from the phonon distribution. This is only possible in the collective regime, as all the modes share the same MFT. However, in the kinetic-dominated regime this is not possible as the MFT of the different phonons can span several orders of magnitude and the diffusive to ballistic transition has a different time scale for each phonon mode. TL is the generalization of the kinetic transport taking into account the broad distribution of mean free times giving rise to a fractal random walk behavior. This approach properly distinguishes interfacial dynamics from nearby quasiballistic heat flow suppression. In this framework, the effect of different time scales is captured by using the fractal exponent $(\alpha)$ that relates the scaling of the mean-square displacement (MSD) of thermal energy with time. ${ }^{39}$ Considering steady state, the fractal diffusivity $\chi_{\alpha}$ in the TL model is expressed as: ${ }^{31}$

$$
\psi(\xi)=\xi^{2} \frac{\sum_{\mathbf{q}} \frac{C_{v, \mathbf{q}} \Lambda_{\mathbf{q}}{ }^{2}}{\tau_{\mathbf{q}}\left[1+\xi^{2} \Lambda^{2}\right]}}{\sum_{\mathbf{q}} \frac{C_{v, \mathbf{q}}}{1+\xi^{2} \Lambda^{2}}},
$$

where $\psi(\xi) / \xi^{2}$ represents the spacial evolution of the fractal diffusivity $\chi_{\alpha}$.

Fig. 3 represents the spatial transient thermal response for $\mathrm{Si}, \mathrm{In}_{0.53} \mathrm{Ga}_{0.47} \mathrm{As}$ and $\mathrm{Si}_{0.82} \mathrm{Ge}_{0.18}$ obtained from a TL model. ${ }^{31,37}$ It can be seen that the region with a superdiffusive behaviour with fractional exponent spans 4 orders of magnitude in time for $\mathrm{Si}_{0.82} \mathrm{Ge}_{0.18}$ and 3 orders for $\mathrm{In}_{0.53} \mathrm{Ga}_{0.47} \mathrm{As}$. Although for $\mathrm{Si}$ the spatial evolution has not been reported, in the time space the transition from pure ballistic to diffusive regime seems to be gradual without a dominant fractal exponent. ${ }^{31}$ This behavior may be explained in terms of the different weight of the collective contribution $\Sigma$ shown in Fig. 1c. The reasoning is the following. The fractal behavior is a kinetic phenomenon and, as the collective contribution increases, the superdiffusive transition decreases until a single scale appears in the pure collective regime. The spatial/temporal window where superdiffusive transport is observable seems to be directly related to the dominant scattering mechanisms.

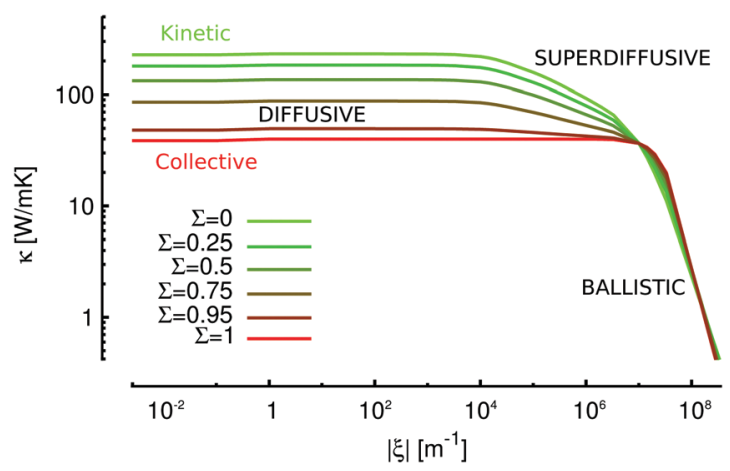

Fig. 4 Fractal thermal conductivity reproduced in the KCM framework for silicon at $300 \mathrm{~K}$. 
The effect of the Lévy flights reproduced from the KCM framework is represented in Fig. 4. As can be observed, while the pure kinetic regime (top green line) shows a clear superdiffusive region, in the collective regime there is a single transition scale. When the collective contribution increases (i.e. $\Sigma$ increases) the fractal slope becomes smoother until it disappears for $\Sigma=1$. Notice that the value of $\Sigma=0.5$ corresponds approximately to silicon.

Using KCM in combination with TL to obtain the phonon MFP spectral distribution from experiments should include the collective effect in the functions used to convolute with experimental results, usually called suppression functions. The obtained function should present in this case an step function at its corresponding scale. In Fig. 5 the predicted MFP accumulation functions by KCM for $\mathrm{Si}, \mathrm{In}_{0.53} \mathrm{Ga}_{0.47} \mathrm{As}$, and $\mathrm{Si}_{0.82} \mathrm{Ge}_{0.18}$ are shown. The jump in cumulative thermal conductivity can be seen clearly for silicon at the MFP of $\sim 20 \mathrm{~nm}$. In contrast, the contribution of the collective regime to thermal conductivity in the alloys is almost negligible. Accordingly, a definite slope can be observed in the thermal conductivity accumulation function (TCAF). Notice that this does not mean that $\mathrm{N}$ scattering is not important as kinetic slowdown is still present.

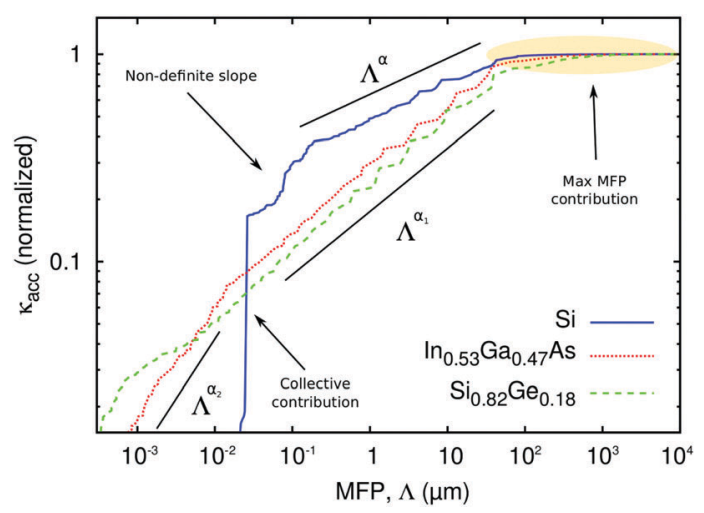

Fig. 5 Thermal conductivity accumulation function (TCAF) of natural $\mathrm{Si}$, $\mathrm{Si}_{0.82} \mathrm{Ge}_{0.18}$ and $\mathrm{In}_{0.53} \mathrm{Ga}_{0.47}$ As at $300 \mathrm{~K}$ with $L_{\text {eff }}=7 \mathrm{~mm}$.

As noticed by Vermeersch et al.,$^{31}$ the slope of the accumulation function can be related to the exponent of the dominant scattering mechanism. To see this relation, Fig. 6 shows the $U$ and impurity/ alloy relaxation times for $\mathrm{Si}, \mathrm{In}_{0.53} \mathrm{Ga}_{0.47} \mathrm{As}$, and $\mathrm{Si}_{0.82} \mathrm{Ge}_{0.18}$. It can be observed that $\mathrm{U}$ scattering is dominant for silicon, which has a trend that goes from $\tau \propto \omega^{-3}$ to $\tau \propto \omega^{-2}$, according to the first principles calculations and in agreement with Han's expressions. ${ }^{40}$ For $\mathrm{Si}_{0.82} \mathrm{Ge}_{0.18}$ and $\mathrm{In}_{0.53} \mathrm{Ga}_{0.47}$ As the dominant scattering is the alloy with $\tau \propto \omega^{-4}$, although is stronger in $\mathrm{Si}_{0.82} \mathrm{Ge}_{0.18}$. This shows why both have also the same exponent in the accumulation function but with different extents of the superdiffusive region.

In addition, from Fig. 6 it can be obtained an explanation of why the superdiffusive window is narrower for $\operatorname{In}_{0.53} \mathrm{Ga}_{0.47} \mathrm{As}$ than for $\mathrm{Si}_{0.82} \mathrm{Ge}_{0.18}$. According to Vermeersch et al. ${ }^{31}$ the fractal exponent can be defined only when the dominant relaxation time has an exponent $n \geq 3$. Having a look at the dominant relaxation times, the narrowest window will correspond to

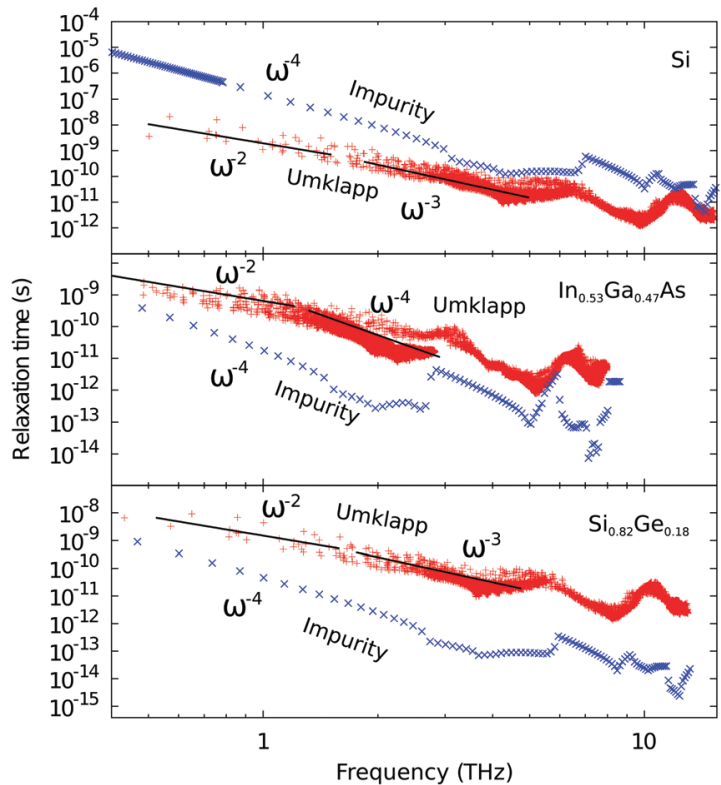

Fig. 6 Umklapp and impurity/alloy first principles relaxation times for $\mathrm{Si}$, $\ln _{0.53} \mathrm{Ga}_{0.47} \mathrm{As}$ and $\mathrm{Si}_{0.82} \mathrm{Ge}_{0.18}$ at $300 \mathrm{~K}$.

silicon, where only the central part of the spectrum has $n=3$. Comparing between the two alloys, it can be observed that for $\mathrm{In}_{0.53} \mathrm{Ga}_{0.47} \mathrm{As}$ at the left side of the plot around $0.5 \mathrm{THz}$ the relaxation time with $\tau \propto \omega^{-2}$ starts to be important in front of $\tau \propto \omega^{-4}$, while for $\mathrm{Si}_{0.82} \mathrm{Ge}_{0.18}$ is not yet relevant. Therefore when $\tau \propto \omega^{-2}$ a fractal dimension is not defined. Notice that this effect can be only clarified if the low frequency relaxation times are well interpreted, as done in the present work by using Han's expressions, ${ }^{40}$ and are examined independently without considering $\mathrm{N}$ scattering as resistive. The effect of $\mathrm{N}$ scattering does not change the superdiffusive window but only its slope trough $\Sigma$ as shown in Fig. 4.

In conclusion, we have analyzed the collective contribution to thermal transport in alloys, which is a consequence of the role of normal phonon scattering. First, thermal conductivity values derived from the Kinetic Collective Model for $\mathrm{Si}_{1-x} \mathrm{Ge}_{x}$ and $\operatorname{In}_{x} \mathrm{Ga}_{1-x} \mathrm{As}$ alloys show good agreement with experimental data and previous calculations. Without introducing any fitting parameters, we have shown that the proper combination of kinetic and collective transport due to the normal processes can accurately predict thermal conductivity at low alloy concentrations. Impurity concentrations as little as $0.4 \%$ almost eliminate the collective contribution in $\mathrm{Si}_{1-x} \mathrm{Ge}_{x}$, while in $\mathrm{In}_{x} \mathrm{Ga}_{1-x} \mathrm{As}$, the collective contribution is strongly reduced, but not completely removed, at $4 \%$ concentrations. This insight could be useful when addressing phenomena such as phonon drag or dopant effects in semiconductor thermoelectrics. Secondly, while the impact of collective transport on steady-state thermal conductivity of bulk alloys is negligible at most concentrations, the role of normal scattering is always important by slowing down the kinetic transport, thus reducing thermal conductivity by $4-15 \%$ at room temperature. The latter can have a big impact on the analysis of ultrafast thermal transport at small length scales. Finally, from the 
phonon spectral analysis, it is shown that the collective regime, where phonons share the same mean free path, narrows the time window of superdiffusive heat transport. This reduction is visible in highly pure samples such as silicon. In addition, the slope of the accumulation function can been correlated to the dominant scattering mechanism in both kinetic and collective regimes.

\section{Conflicts of interest}

There are no conflicts to declare.

\section{Acknowledgements}

This work has been partially funded by the Spanish Ministerio de Economía y Competitividad under Project Nos TEC2012-31330, FIS2012-32099, MAT2012-33483, TEC2015-67462-C2-2-R (MINECO/ FEDER), TEC2015-67462-C2-1-R (MINECO/FEDER), Consolider nanoTHERM CSD2010-00044 and Generalitat de Catalunya under Project 2014-SGR-0064 and 2014-SGR-384. Also, the research leading to these results has received funding from the European Union Seventh Framework Programme under grant agreement No. 604391 Graphene Flagship.

\section{References}

$\dagger$ Notice that in this case the predicted thermal conductivity for $\mathrm{Si}$ is $\sim 130 \mathrm{~W} \mathrm{~m}^{-1} \mathrm{~K}^{-1}$, while the current reported experimental value is $\sim 145 \mathrm{~W} \mathrm{~m}^{-1} \mathrm{~K}^{-1} \cdot{ }^{17}$

1 C. De Tomas, A. Cantarero, A. F. Lopeandia and F. X. Alvarez, J. Appl. Phys., 2014, 115, 164314.

2 C. De Tomas, A. Cantarero, A. F. Lopeandia and F. X. Alvarez, Proc. R. Soc. A, 2014, 470, 20140371.

3 L. Lindsay, D. a. Broido and T. L. Reinecke, Phys. Rev. B: Condens. Matter Mater. Phys., 2013, 87, 165201.

4 A. Ward and D. A. Broido, Phys. Rev. B: Condens. Matter Mater. Phys., 2010, 81, 085205.

5 D. A. Broido, M. Malorny, G. Birner, N. Mingo and D. A. Stewart, Appl. Phys. Lett., 2007, 91, 231922.

6 P. Torres, A. Torello, J. Bafaluy, J. Camacho, X. Cartoixà and F. X. Alvarez, Phys. Rev. B, 2016, 95, 165407.

7 S. Lee, D. Broido, K. Esfarjani and G. Chen, Nat. Commun., 2015, 6, 6290.

8 K. M. Hoogeboom-Pot, J. N. Hernandez-Charpak, X. Gu, T. D. Frazer, E. H. Anderson, W. Chao, R. W. Falcone, R. Yang, M. M. Murnane, H. C. Kapteyn and D. Nardi, Proc. Natl. Acad. Sci. U. S. A., 2015, 112, 201503449.

9 N. Mingo, L. Yang, D. Li and A. Majumdar, Nano Lett., 2003, 3, 1713-1716.

10 M. Kazan, G. Guisbiers, S. Pereira, M. R. Correia, P. Masri, A. Bruyant, S. Volz and P. Royer, J. Appl. Phys., 2010, 107, 083503.

11 R. B. Wilson and D. G. Cahill, Nat. Commun., 2014, 5, 5075.

12 R. E. Peierls, Quantum Theory of Solids, Oxford University Press, 2007.

13 A. Ward, D. A. Broido, D. A. Stewart and G. Deinzer, Phys. Rev. B: Condens. Matter Mater. Phys., 2009, 80, 1-8.
14 A. Cepellotti and N. Marzari, Phys. Rev. X, 2016, 6, 041013. 15 L. Chaput, Phys. Rev. Lett., 2013, 110, 1-5.

16 J. Garg, N. Bonini, B. Kozinsky and N. Marzari, Phys. Rev. Lett., 2011, 106, 1-4.

17 A. V. Inyushkin, A. N. Taldenkov, A. Y. Yakubovsky, A. V. Markov, L. Moreno-Garsia and B. N. Sharonov, Semicond. Sci. Technol., 2003, 18, 685-688.

18 P. Torres, Kinetic Collective Model: BTE-based hydrodynamic model for thermal transport, 2017, accessed August 20, 2017, https://physta.github.io/.

19 L. Bellaiche and D. Vanderbilt, Phys. Rev. B: Condens. Matter Mater. Phys., 2000, 61, 7877-7882.

20 P. Giannozzi, S. Baroni, N. Bonini, M. Calandra, R. Car, C. Cavazzoni, D. Ceresoli, G. L. Chiarotti, M. Cococcioni, I. Dabo, A. Dal Corso, S. de Gironcoli, S. Fabris, G. Fratesi, R. Gebauer, U. Gerstmann, C. Gougoussis, A. Kokalj, M. Lazzeri, L. Martin-Samos, N. Marzari, F. Mauri, R. Mazzarello, S. Paolini, A. Pasquarello, L. Paulatto, C. Sbraccia, S. Scandolo, G. Sclauzero, A. P. Seitsonen, A. Smogunov, P. Umari and R. M. Wentzcovitch, J. Phys.: Condens. Matter, 2009, 21, 395502. 21 P. Hohenberg and W. Kohn, Phys. Rev., 1964, 136, 864-871. 22 W. Kohn and L. J. Sham, Phys. Rev., 1965, 140, A1133.

23 J. P. Perdew and A. Zunger, Phys. Rev. B: Condens. Matter Mater. Phys., 1981, 23, 5048-5079.

24 U. von Barth and R. Car, Unpublished notes, 1977.

25 R. R. A. D. Corso, S. Baroni and S. de Gironcoli, Phys. Rev. B: Condens. Matter Mater. Phys., 1993, 47, 3588-3592.

26 G. Nilsson and G. Nelin, Phys. Rev. B: Solid State, 1972, 6, 3777-3786.

27 D. Strauch and B. Dorner, J. Phys.: Condens. Matter, 1990, 2, 1457.

28 B. Abeles, D. Beers, G. Cody and J. Dismukes, Phys. Rev., 1962, 125, 44-46.

29 J. P. Dismukes, L. Ekstrom, E. F. Steigmeier, I. Kudman and D. S. Beers, J. Appl. Phys., 1964, 35, 2899-2907.

30 M. C. Steele and F. D. Rosi, J. Appl. Phys., 1958, 29, 1517-1520.

31 B. Vermeersch, J. Carrete, N. Mingo and A. Shakouri, Phys. Rev. B: Condens. Matter Mater. Phys., 2015, 91, 085202.

32 H. Casimir, Physica, 1938, 5, 495-500.

33 P. G. Klemens, Proc. Phys. Soc., London, Sect. A, 1955, 68, 1113-1128.

34 W. Capinski, H. Maris and S. Tamura, Phys. Rev. B: Condens. Matter Mater. Phys., 1999, 59, 10105-10110.

35 P. Mycock, Solid-State Electron., 1966, 10, 161-168.

36 W. Kim, J. Zide, A. Gossard, D. Klenov, S. Stemmer, A. Shakouri and A. Majumdar, Phys. Rev. Lett., 2006, 96, 1-4.

37 B. Vermeersch, A. M. S. Mohammed, G. Pernot, Y. R. Koh and A. Shakouri, Phys. Rev. B: Condens. Matter Mater. Phys., 2015, 91, 085203.

38 J. C. Maxwell, Philos. Mag., 1868, 35, 49-88.

39 B. Vermeersch, A. M. S. Mohammed, G. Pernot, Y. R. Koh and A. Shakouri, Phys. Rev. B: Condens. Matter Mater. Phys., 2014, 90, 1-5.

40 Y.-J. Han, Phys. Rev. B: Condens. Matter Mater. Phys., 1996, 54, 8977-8980. 